\title{
Reduction of the prediction horizon of predictive energy management for a plug-in HEV in hilly terrain
}

\author{
B. Bader ${ }^{1}$, O. Torres ${ }^{1}$, J. A. Ortega ${ }^{1}$, G. Lux $^{2}$ \& J. L. Romeral ${ }^{1,3}$ \\ ${ }^{1}$ MCIA, Technical University of Catalonia (UPC), Spain \\ ${ }^{2}$ SEAT Technical Centre, Spain \\ ${ }^{3}$ CTM Technical Centre, Spain
}

\begin{abstract}
Due to the great weight and high costs of electric energy storage systems (ESS), the number of pure electric vehicles $(\mathrm{EV})$ is increasing only slowly. As a compromise between the autonomous hybrid electric vehicle (HEV) and EV, the plug-in HEV (PHEV) allows, like the EV, the recharging of the battery by the grid but brings also a combustion engine so as not to depend on the limited electric range of the vehicle. Next to the sizing of the vehicle components, the energy management strategy has an important influence on the fuel consumption of the vehicle. To minimize fuel consumption, predictive energy management is necessary, as all stored electric energy should be consumed by the end of the trip. In this way it is possible to minimize fuel consumption by substituting as much fuel as possible by the use of electric energy. In order to reach the global optimal result, a prediction horizon of the optimization for the duration of the entire trip is necessary. However, due to model uncertainties and the limited calculation capacities of the control units in a vehicle the global optimum cannot be achieved. Therefore, measures have to be taken to reduce the computation cost on the one hand and achieve results close the global optimum on the other. One of these measures, next to an adequate optimization algorithm, is the reduction of the prediction horizon. In this study, for a real life cycle including urban and highway parts a variation of the prediction horizon is carried out and the influence on the fuel consumption is simulated. The respective results are calculated using Dynamic Programming to exclude any influence of the chosen
\end{abstract}


energy management strategy. The results are compared to the global optimal fuel consumption of the used driving cycle.

Keywords: plug-in HEV, PHEV, energy management, prediction.

\section{Introduction}

The numbers of sold pure electric vehicles (EV) is still low, as the costs of the battery are still high and the electric range of the vehicles low. This situation together with the long recharging times of the battery compared to a conventional fuel vehicle, hamper the breakthrough of this vehicle type in the near future. Instead, hybrid electric vehicles (HEV) are supposed to fill the gap between these both vehicle types. A further step to more electrification of the vehicles is the plug-in HEV (PHEV), which disposes of a significantly higher battery capacity and the possibility of recharging the battery by means of the electric grid.

Using PHEV on trips longer than the electric range, the use of predictive energy management strategies (EM) will gain importance. While for autonomous HEV the stored electric energy at the end of the trip is of minor importance, for PHEV a higher electric consumption during the trip in general means lower fuel consumption. Therefore, for trips longer than the electric range it should reach the minimal state of charge (SOC) of the battery - in that way, fuel consumption can be minimized [1].

A basic energy management implementation to reach this aim without the necessity of prediction is the all-electric-range strategy (AER) [2]. The AER strategy starts the vehicle in the beginning of the driving cycle in a pure electric driving mode. After reaching a predefined low electric energy level of the battery the energy management switches to charge sustaining mode, which is known from autonomous HEV. In contrary to predictive strategies, AER does not need any knowledge of the future trip. But as consuming the stored electric energy already in the beginning of the trip, it has afterwards less potential to avoid bad efficiency regions of the combustion engine. Secondly, in the first part using electric mode the electric motor is maybe driven is less efficient regions.

Therefore, to minimize fuel consumption, a predictive strategy is needed which distributes the stored electric energy over the whole driving cycle. It has been shown that compared to the AER strategy the fuel consumption can be reduced when distributing the electric energy on the whole driving cycle $[3,4]$.

Due to the limited capacity of the computer on the vehicle, the computational effort of the strategy has to be considered. As it increases with the prediction horizon, a compromise between fuel saving and prediction horizon length has to be found. In [6] a model predictive control with a limited prediction horizon is used for a HEV.

In this paper, the influence of the horizon length is evaluated for a parallel plug-in HEV. To avoid any influence of the control strategy, Dynamic Programming (DP) is used to find the respective optimal behaviour. It is supposed that information about the whole trip length and additionally velocity 
and road slope information about the current prediction section are available to the strategy.

\section{Vehicle structure}

For the simulation the body parameters of the passenger car SEAT Ibiza ST are used, taking into account the weight of the additional components as battery and electric motor. The simulated hybrid drivetrain has a parallel structure, that is the electric motor and combustion engine are mounted on the same drive shaft so that the sum of their torques are applied to the drive shaft. The combustion engine can be separated by a clutch from the electric machine to allow electric driving without the friction losses of the engine (fig. 1).

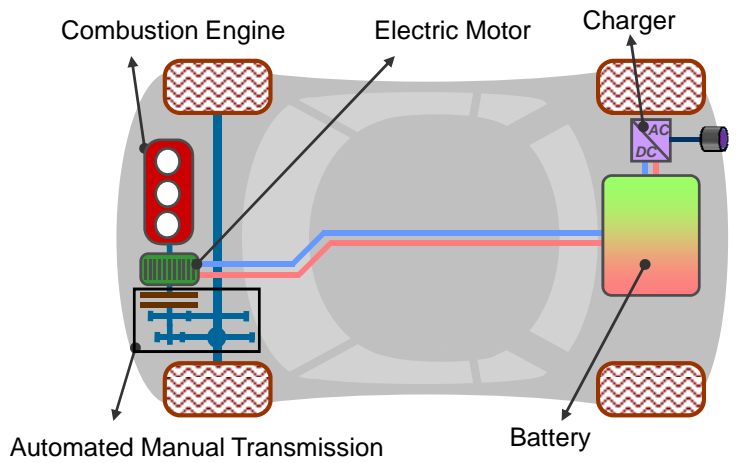

Figure 1: Drivetrain scheme of a parallel PHEV.

The double clutch gearbox disposes of 7 gears to allow the engine working in a high efficiency region. Due to the double clutch principle fast gear shifting is assumed and therefore shifting losses not considered in the model. The battery has a capacity of $4 \mathrm{kWh}$ and can be recharged during the trip by the combustion engine or afterwards by a connection to the electric grid. To avoid damage to the battery the SOC is not allowed to be below of 0.2. To enable at the end of the trip the use of the vehicle in hybrid mode without recharging, the strategy leaves $1.2 \mathrm{kWh}$ (which corresponds with a SOC of 0.3 ) at the trip end in order to make it possible to use a HEV charge sustaining operation mode. This might be necessary as there is not always enough time for recharging before the next trip.

Table 1: $\quad$ Vehicle simulation parameters.

\begin{tabular}{|l|l|l|l|}
\hline Vehicle Mass & $1450 \mathrm{~kg}$ & $\mathrm{~T}_{\text {EM,max }}$ & $250 \mathrm{Nm}$ \\
\hline $\mathrm{A}_{\mathrm{f}}$ & $2.2 \mathrm{~m}^{2}$ & $\mathrm{E}_{\text {battery }}$ & $4 \mathrm{kWh}$ \\
\hline Gears & 7 & $\mathrm{E}_{\text {battery,min }}$ & $0.8 \mathrm{kWh}$ \\
\hline $\mathrm{P}_{\text {ICE,max }}$ & $51 \mathrm{~kW}$ & $\mathrm{~V}_{\text {battery }}$ & $300 \mathrm{~V}$ \\
\hline $\mathrm{T}_{\text {ICE,max }}$ & $110 \mathrm{Nm}$ & $\mathrm{R}_{\text {battery }}$ & $352.5 \mathrm{~m} \Omega$ \\
\hline $\mathrm{P}_{\mathrm{EM}, \max }$ & $40 \mathrm{~kW}$ & & \\
\hline
\end{tabular}




\section{Driving cycles}

One drawback of governmental driving cycles as the FTP7x or the NEDC for the evaluation of HEV is the lack of slope information. Therefore, to take into account the influence of hilly terrain, a real life cycle is used. Two recorded driving cycles are used, from Barcelona to the SEAT Technical Centre in Martorell and the return trip. The return trip is not exactly the same route and is driven with higher speed. The length is about 33km and the trips last 1903s and 2231s (figs 2 and 3).It consists of a short urban part at the beginning and a longer one at the end. During the highway part the velocity increases up to $115.1 \mathrm{~km} / \mathrm{h}$. The maximal slope is $4 \%$. The characteristics of the cycle and the vehicle cause that it is not possible to drive the whole trip in pure electric driving mode, but that the combustion engine has to be used.

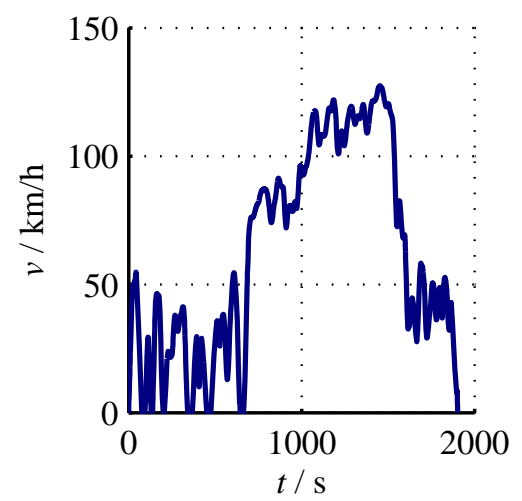

Figure 2: Vehicle velocity of the Figure 3: driving cycle BCN-CTS.

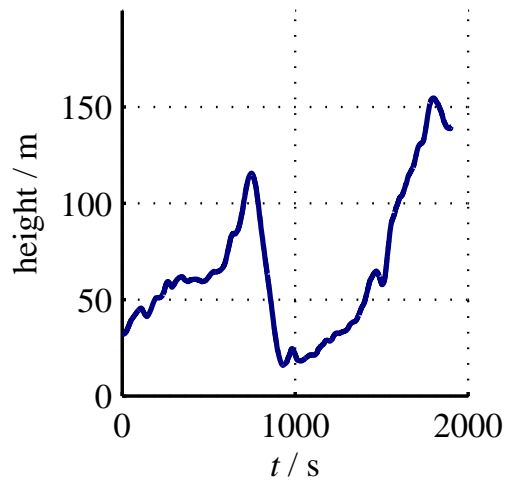

Height profile of the driving cycle BCN-CTS.

Due to the height difference of $122 \mathrm{~m}$ between start and end point the vehicle benefits in the cycle CTS-BCN of the change of its potential energy. This energy change is

$$
\Delta E_{p o t}=m g \Delta h \approx 1.71 \mathrm{MJ} \approx 0.47 \mathrm{kWh} .
$$

Compared to the available electric energy of $2.6 \mathrm{kWh}$ this is a non-negligible amount and will therefore later be considered in the energy management. The characteristics of both driving cycles are summarized in the table below (table 2).

Table 2: $\quad$ Characteristics of simulated driving cycles.

\begin{tabular}{|l|l|l|l|l|l|}
\hline Cycle & Duration/s & Length/km & $\mathrm{v}_{\max } / \mathrm{km} / \mathrm{h}$ & $\mathrm{v}_{\mathrm{avg}} / \mathrm{km} / \mathrm{h}$ & $\Delta \mathrm{h} / \mathrm{m}$ \\
\hline BCN-CTS & 1903 & 33.1 & 127.4 & 62.6 & 107.6 \\
\hline CTS-BCN & 2231 & 33.3 & 115.1 & 53.8 & 122.1 \\
\hline
\end{tabular}




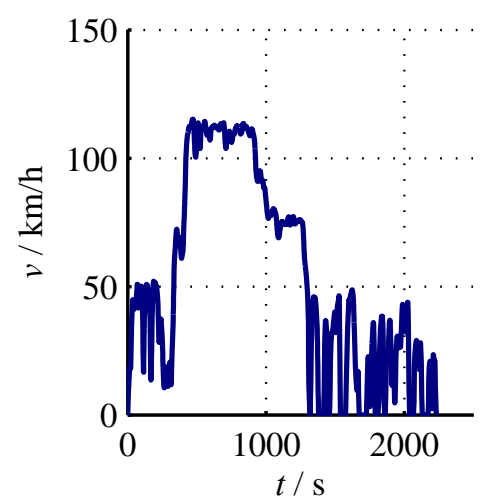

Figure 4: Vehicle velocity of the Figure 5: driving cycle CTS-BCN.

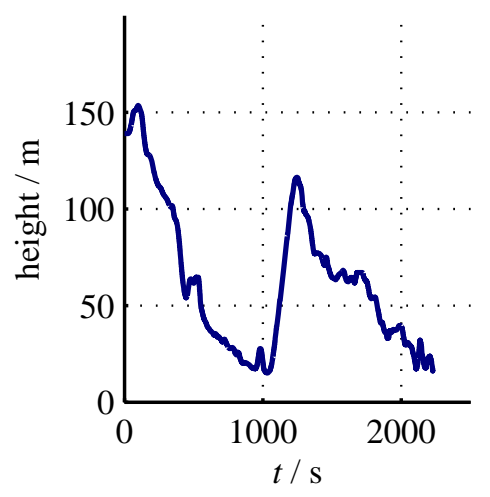

Height profile of the driving cycle CTS-BCN.

\section{Vehicle models}

The vehicle model used in the simulation is modelled in the environment Modelica/Dymola. In order to obtain a sufficiently quick optimization, the optimization with Dynamic Programming takes place on a stationary backward model, which does not contain time dependent elements. The model is validated with measurement data from a roller test bench. In the following the different model components are shortly described.

\subsection{Combustion engine}

The fuel consumption of the combustion engine is modelled by a measured consumption map of a $51 \mathrm{~kW}$ engine. The calculated revolution number and the torque demand are used to interpolate the corresponding fuel consumption.

$$
m_{f}=f\left(T_{I C E}, \omega_{I C E}\right)
$$

where $T_{I C E}$ is the engine torque and $\omega$ the angular velocity of the engine shaft.

\subsection{Electric traction chain}

For the Electric Motor the electric losses are modelled by an electric losses map. Similar to the combustion engine the revolution number and the torque are used to interpolate the corresponding electric losses and so the electric power input is known.

$$
P_{\text {electric }}=P_{\text {mech }}+f_{\text {losses }}\left(T_{E M}, \omega_{E M}\right)
$$

The battery is modelled using discrete elements of the internal resistances. For the inverter a constant efficiency of $92 \%$ is assumed. 


\section{Energy management}

The energy management controls the energy content of the battery during the trip. This is done by the change of the torque distribution of the demanded torque by the driver to the electric motor and the combustion engine. In this way the change between the different operating modes recuperation, electric, boost and charge is controlled. For plug-in HEV on trips longer than the electric range it is wished to substitute as much fuel energy by electric energy to minimize fuel consumption. That is, when starting the trip with $\mathrm{SOC}_{\max }$, at the end of the cycle there should remain $\mathrm{SOC}_{\min }$ with a before defined cushion to allow to use the vehicle in charge sustaining mode. The initial SOC of the battery is defined as 0.9 . This, with a minimal SOC of 0.2 , leads to an electric energy of $E_{\text {battery }}=$ $2.6 \mathrm{kWh}$ which can be consumed over the trip length.

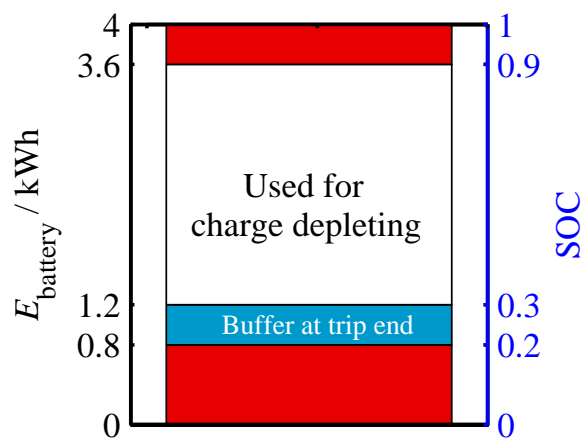

Figure 6: Electric energy used during the charge depleting mode (CD) and the buffer reserved for charge sustaining mode (CS) at the end of the cycle.

\subsection{Dynamic programming}

Dynamic Programming (DP) is a common algorithm to calculate the global optimal solution of a discrete stochastic optimization task. However, as here the optimization task is assumed to be not stochastic, the resulting problem is a shortest way problem [5].

As it can be applied only on a discrete optimization task, a discretization grid for the time and the states of the model has to be defined. A finer grid means higher exactness but lower computation speed. As the algorithm is considering all possible solutions of the problem, the computation cost is quite high. Furthermore, a simple problem has to be defined as the computation cost increases exponentially with the states of the optimization problem. Especially time dependent states are problematic, as the computation cost increase exponentially with the number of states. 


\subsubsection{Algorithm}

The system to which the optimization is applied can be generally be described by

$$
x_{k+1}=f_{k}\left(x_{k}, u_{k}\right)
$$

where $x_{k}$ and $u_{k}$ are the state and the control variable at time step $k$. Here the control variable is the torque of the electric machine $T_{E M}$ while the state variable is the SOC of the battery.

The control problem can be described as finding the optimal control sequence

$$
\pi^{o}=\{u(1), u(2), \ldots . u(N)\}
$$

which minimizes

$$
J^{o}\left(x_{o}\right)=\min _{\pi \in \Pi} J_{\pi}\left(x_{o}\right)
$$

where $\Pi$ is the set of all possible control sequences, $J$ the sum of the fuel consumption at every time step and $x_{0}$ the system state at time step $k=0$.

\subsection{Reduced prediction horizon}

To reach the SOC of 0.3 at the trip end, a predictive energy management has to be used. The optimization with a prediction horizon of the whole trip length has two shortcomings. Firstly, the computational effort rises significantly subject to the used optimization algorithm. Using shortest way optimization like DP, the effort increases more than proportional to the horizon time. Secondly, using online optimization strongly simplified drive train models have to be deployed, thus the inexactness is increasing with the prediction horizon. Therefore, shorter prediction horizons can be advantageous. Using shorter prediction horizons means to start the optimization repeatedly for the next trip section. In this paper, the optimization is repeated every 120s during the trip. Thus the on-board computer has 120 s to finish the optimization for the next prediction horizon. A difficulty of a shorter prediction horizon is to define the final SOC value at the end of every optimization step, as for PHEV a predefined SOC at the trip end is to be reached. Here, a SOC set curve is precalculated, from which the optimization algorithm gets the SOC set point at the end point of the prediction horizon. That is, starting at $\mathrm{t}=0 \mathrm{~s}$ with a prediction horizon of $600 \mathrm{~s}$, the algorithm gets the SOC to be reached at $\mathrm{t}=600 \mathrm{~s}$ from that function. This step is repeated every 120s, that is at $t=120$ s the algorithm gets from the curve the SOC set point of $t=720$ s and starts the optimization again. To evaluate the influence of the precalculated function, three different SOC curves are implemented. The first one is linear over the trip distance (fig. 7), starting at 0.9 and declining to 0.3 at the trip end. Converting the function to the time base, the function loses its linear appearance (fig. 8). 


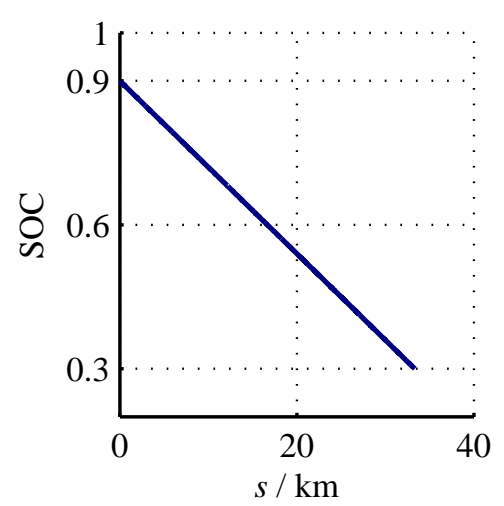

Figure 7: Linear SOC set point Figure 8: Linear SOC set point function over distance for cycle CTS-BCN.

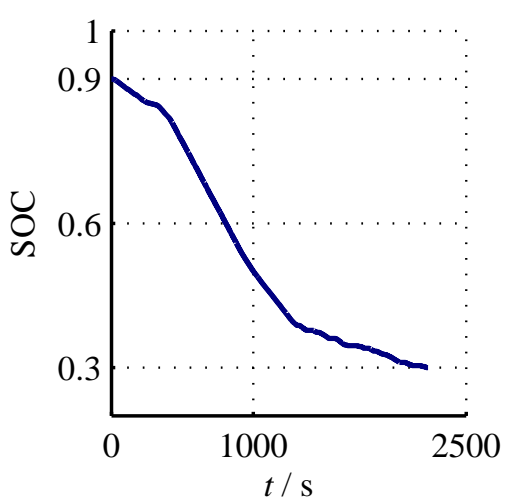

function over time for cycle CTS-BCN.

The second curve (curve B, fig. 9 and fig. 10) takes into account also the road slope. In a first step the total available energy besides the energy generated by the combustion engine is calculated, that is the available electric energy from the battery (60\%) and the potential energy due to the height difference.

$$
E_{\text {total }}=\Delta E_{\text {battery }}+\Delta E_{\text {pot }}=0.6 E_{\text {battery }}+\Delta E_{\text {pot }}
$$

This energy is distributed linearly over the trip and adapted to the current change during the trip

$$
\operatorname{SOC}(s)=S O C_{\text {init }}-\frac{E_{\text {total }}}{E_{\text {battery }}} \frac{s}{s_{\text {total }}}-\Delta E_{\text {pot }}
$$

The third SOC (curve C, fig. 9 and fig. 10)) curve also takes into account the efficiency losses of the transmission. To do so, in eqn. (5) and eqn. (6) $\Delta E_{p o t}$ is substituted by

$$
\Delta E_{\text {pot }}^{C}=\Delta E_{\text {pot }} \eta_{\text {transmission }}
$$

The curves B and C differ significantly from the only linear curve A. During the first inclination of the cycle BCN-CTS the functions B, C drop faster, as it is considered more energy is necessary (fig.9). On the other hand, during the following downhill grade the function $\mathrm{B}, \mathrm{C}$ even increases, as the recuperation energy or the less energy necessity of the vehicle is considered. The same pattern can be seen for the cycle CTS-BCN (fig. 10). So is in the last part the decrease very slow, as less energy consumption because of the negative slope is assumed. 


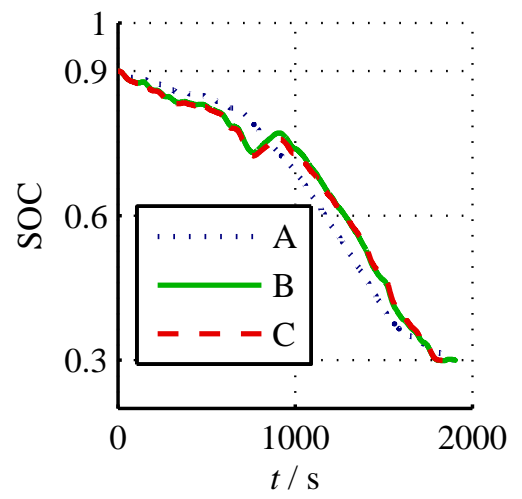

Figure 9: $\quad$ Predefined SOC set point functions for cycle BCNCTS.

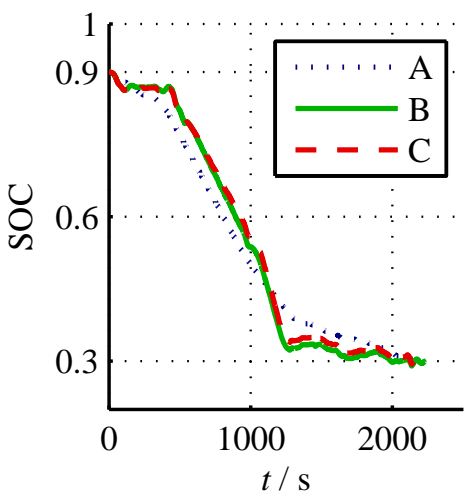

Figure 10: Predefined SOC set point functions for cycle CTS-BCN.

\section{Simulation results}

The simulations are done using the three different SOC set point functions A, B, C with prediction horizon lengths of 300, 480, 600 and 1200s. The simulations are repeated for both driving cycles. As reference the global optimum of both cycles is computed by using a prediction horizon length of the whole trip.

For the cycle BCN-CTS the fuel consumption of a prediction horizon of 1200 s is for all SOC functions almost equal to the global optimum (fig. 11). Reducing the horizon to 600s, the consumption increases very slightly from $0.12 \%$ to $0.24 \%$. A further reduction to 480 s still gives good results with a maximal deviation of $0.34 \%$. A horizon of 300 s gives a maximal deviation of $0.56 \%$. The SOC functions using road slope information always outdo function A. The desired final of 0.3 is reached with all SOC functions. The used SOC function shows only small influence to the results, especially between the both function using height information.

The results of the return trip CTS-BCN differ from the results above. The difficulty of this cycle is that in the last urban part the road slope is negative, thus the power demand during this part is low. Using SOC function A, the energy management cannot consume all electric energy by the cycle end. Therefore, the final SOC value is above 300s (table 3). The remaining electric energy in the battery leads to a higher fuel consumption, as instead of the electric energy fuel was used to generate the demanded torque. 


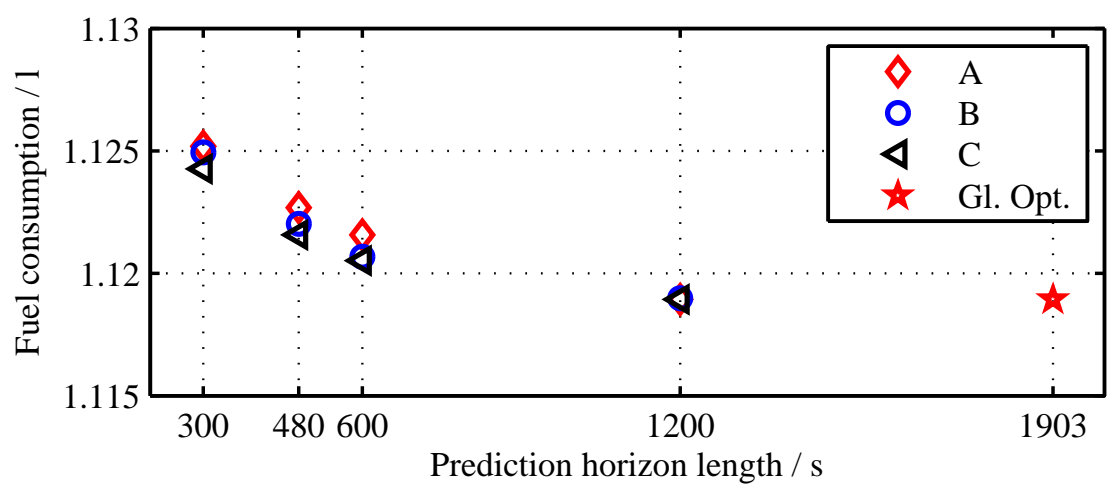

Figure 11: Fuel consumption of trip BCN-CTS.

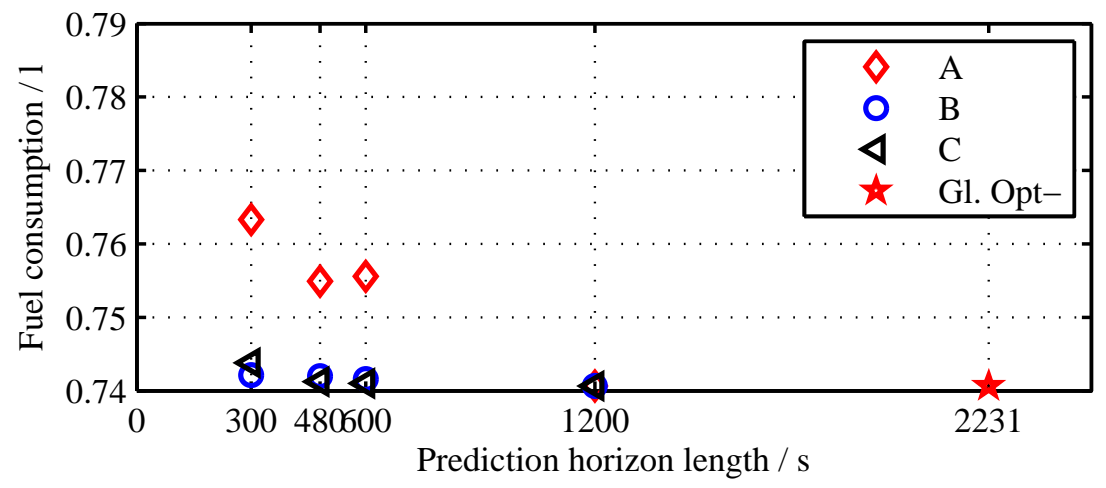

Figure 12: Fuel consumption of trip CTS-BCN.

Table 3: $\quad$ Fuel consumption using the SOC function A.

\begin{tabular}{|l|l|l|l|l|l|l|}
\hline & \multicolumn{3}{|c|}{ BCN-CTS } & \multicolumn{3}{c|}{ CTS-BCN } \\
\hline $\begin{array}{l}\text { Prediction } \\
\text { Horizon/s }\end{array}$ & Fuel/l & SOC $_{\text {end }}$ & Deterioration/\% & Fuel// & SOC & \\
end & Deterioration/\% \\
\hline 300 & 1.125 & 0.3 & 0.56 & 0.763 & 0.3236 & 3.05 \\
\hline 480 & 1.123 & 0.3 & 0.34 & 0.755 & 0.3151 & 1.92 \\
\hline 600 & 1.122 & 0.3 & 0.24 & 0.756 & 0.3158 & 2.01 \\
\hline 1200 & 1.119 & 0.3 & 0 & 0.741 & 0.3 & 0 \\
\hline Full Trip & 1.119 & 0.3 & 0 & 0.741 & 0.3 & 0 \\
\hline
\end{tabular}


Table 4: $\quad$ Fuel consumption using the SOC function B.

\begin{tabular}{|l|c|c|c|l|l|l|}
\hline & \multicolumn{3}{|c|}{ BCN-CTS } & \multicolumn{3}{c|}{ CTS-BCN } \\
\hline $\begin{array}{l}\text { Prediction } \\
\text { Horizon/s }\end{array}$ & Fuel/l & SOC $_{\text {end }}$ & Deterioration/\% & Fuel/l & SOC & \\
\hline 300 & 1.125 & 0.3 & 0.53 & 0.742 & 0.3 & Deterioration/\% \\
\hline 480 & 1.122 & 0.3 & 0.28 & 0.742 & 0.3 & 0.20 \\
\hline 600 & 1.121 & 0.3 & 0.16 & 0.742 & 0.3 & 0.13 \\
\hline 1200 & 1.119 & 0.3 & 0 & 0.741 & 0.3 & 0 \\
\hline
\end{tabular}

Table 5: $\quad$ Fuel consumption using the SOC function C.

\begin{tabular}{|l|l|l|l|l|l|l|}
\hline & \multicolumn{3}{|c|}{ BCN-CTS } & \multicolumn{3}{c|}{ CTS-BCN } \\
\hline $\begin{array}{l}\text { Prediction } \\
\text { Horizon/s }\end{array}$ & Fuel/1 & SOC $_{\text {end }}$ & Deterioration/\% & Fuel/l & SOC $_{\text {end }}$ & Deterioration/\% \\
\hline 300 & 1.124 & 0.3 & 0.48 & 0.744 & 0.3024 & 0.42 \\
\hline 480 & 1.122 & 0.3 & 0.24 & 0.741 & 0.3 & 0.07 \\
\hline 600 & 1.121 & 0.3 & 0.12 & 0.741 & 0.3 & 0.04 \\
\hline 1200 & 1.119 & 0.3 & 0 & 0.741 & 0.3 & 0 \\
\hline
\end{tabular}

\section{Conclusions}

Using simple SOC set curves, the prediction horizon can be reduced to several minutes while deteriorating the fuel consumption only slightly. For both driving cycles the prediction horizon length of 1200 s and the global optimal results are not notable. Using shorter prediction horizons, the difference of the fuel consumption to the global optimum becomes notable. Reducing the prediction horizon to 600s, the fuel consumption only increases by up to $2.01 \%$. On the other hand, this happens mainly because not all stored electric energy is consumed and can be avoided including rode slope information to the set point function.

Considering the results with function $\mathrm{B}, \mathrm{C}$, the only slight increase shows that for optimal behaviour of the engine and electric motor it is not necessary to optimize over long horizons. Therefore, the fuel consumption of PHEV can be optimized while reaching a desired SOC value at the trip end without the necessity of an optimization over the whole trip.

\section{Acknowledgements}

VERDE is a CENIT project funded by the Ministry of Economy and Competitiveness of the Spanish Government. It is led by SEAT, coordinated by CTM Centre Tecnològic and integrated by the following companies: AIA, Cegasa, Circutor, Cobra, Endesa, Ficosa, Green Power, Iberdrola, Infranor, Lear, Mapro, Red Eléctrica España, Siemens, Rovalma and Tècnicas Reunidas; and Research Institutes: AICIA, ASCAMM, CIDETEC, CIRCE, CNM del CSIC, CTM Centre Tecnològic, IIC, IIT de la Universidad Pontificie de Comillas, 
IREC, Leitat, Tecnalia, Universitat Politècnica Catalunya (UPC) and Universidad Carlos III.

\section{References}

[1] S. Stockar, V. Marano, M. Canova, G. Rizzona, L. Guzzella, EnergyOptimal Control of Plug-in Hybrid Electric Vehicles for Real-World Driving Cycles, IEEE Transactions on Vehicular Technology, No. 7, Vol. 60, pp. 2949-2962, 2011

[2] P. Sharer, A. Rousseau, Plug-in Hybrid Electric Vehicle Control Strategy: Comparison between EV and Charge-Depleting Options, SAE International, Warrendale, 2008

[3] Q. Gong, Y. Li, Z.R. Peng, Trip-based Optimal Power Management of Plugin Hybrid Electric Vehicles, IEEE Transaction on Vehicular Technology, No. 6, Vol. 57, pp. 3393-3401, 2008

[4] J. Gonder et al., Energy management strategies for plug-in hybrid electric vehicles, SAE International, No. 1, pp. 290, 2007

[5] D.P. Bertsekas, Dynamic Programming and Optimal Control, Belmont, USA, 2005

[6] V. Ngo, T. Hofmanm M. Steinbuch, Predictive Gear Shift Control for a Parallel Hybrid Electric Vehicle, Vehicle Power and Propulsion Conference (VPPC), Chicago, 2011 